\title{
EROSÃO REAL E ESTIMADA ATRAVÉS DA RUSLE EM ESTRADAS DE USO FLORESTAIS, EM CONDIÇÕES DE RELEVO PLANO A SUAVE ONDULADO
}

\author{
Carla Maria Camargo Corrêa ${ }^{1}$, Renato Antonio Dedecek ${ }^{2}$ \\ ${ }^{1}$ Eng $^{\mathrm{a}}$. Florestal, Dra ${ }^{\mathrm{a}}$., Depto. de Ciências Florestais, UFPR, Curitiba, PR, Brasil - camargocorrea@ ufpr.br \\ ${ }^{2}$ Eng. Agrônomo, Ph.D., EMBRAPA Florestas, Colombo, PR, Brasil - dedecek@cnpf.embrapa.br \\ Recebido para publicação: 03/10/2007 - Aceito para publicação: 05/09/2008
}

\begin{abstract}
Resumo
Este trabalho teve por objetivo identificar as perdas reais de solo provenientes de trechos de estradas de uso florestal e de suas áreas adjacentes, e comparar com as estimativas obtidas através da Revised Universal Soil Loss Equation (RUSLE). A avaliação das perdas de solo teve início logo após a colheita de madeira e se manteve por um ano, com coletas semanais de solo e em casos de chuva com alta intensidade. As condições de relevo variam de plano a suave ondulado, com caracterização edáfica do tipo Argissolo Vermelho-Escuro álico e Argissolo Vermelho-Escuro Latossólico. Para a adequação da RUSLE, foram considerados os seguintes fatores: erosividade da chuva, obtida através de pluviogramas e cruzados com os dados de pluviosidade da empresa; erodibilidade do solo, calculada em função dos resultados de análises de solo provenientes das áreas; declividade e comprimento de rampa, medidos em campo; e fatores $\mathrm{C}$ e $\mathrm{P}$, estipulados através de literatura. Os resultados obtidos evidenciaram significância estatística e alta correlação existente entre os valores reais, mensurados através de calhas coletoras de sedimentos, e os valores estimados através da RUSLE. As perdas de solo provenientes dos trechos de estrada avaliados corresponderam a $99 \%$ das perdas totais observadas no experimento.
\end{abstract}

Palavras-chave: Erosividade; erodibilidade; declividade e comprimento de rampa.

\begin{abstract}
Real and estimative erosion through Rusle from forest roads in plain to soft undulated relief. This work aimed to identify the real soil losses proceeding from segments of forest roads and your adjacent areas, and to compare with estimative obtained through the Universal Revised Soil Loss Equation (RUSLE). The evaluation of the soil losses had beginning soon after the wood harvesting and prorogate per one year, with weekly soil samples, or in rain cases with high intensity. The relief conditions vary from plain to gentle slope, with edafic characterization of the type: Álic Dark-Red Argissols and Dark-Red Argissols Latossolic. For the adequacy of the RUSLE the following factors had been considered: erosivity of the rain, obtained through pluviograms and crossing with the data of rainfall of the company; erodibility of the ground, calculated in function of the results of soil analyzes proceeding from the areas; the length and slope inclination had been measured in field; and factors $\mathrm{C}$ and $\mathrm{P}$, stipulated through literature. The gotten results had evidenced significance statistics and high correlation between the real values, obtained through sediment flume collector and estimated values through the RUSLE. The soil losses proceeding from the segments of forest roads evaluated had corresponded $99 \%$ of the observed total soil losses in the experiment.
\end{abstract}

Keywords: Erosivity; erodibility; length and steepness slope.

\section{INTRODUÇÃO}

A erosão do solo aparece como um problema central no diagnóstico ambiental em estradas de uso florestal, que apresenta como consequências o assoreamento e a poluição de mananciais, além de problemas relacionados à eficiência no escoamento de madeira. As principais causas relacionadas à erosão em estradas de uso florestal ocorrem em função do tráfego intenso e pesado de máquinas e equipamentos durante a colheita de madeira, que exige um planejamento adequado de uma rede viária 
complementar, a fim de se estabelecer a menor densidade possível, mas que atenda as necessidades de logística e transporte florestal.

Alguns parâmetros técnicos relacionados à rede viária de uso florestal podem interferir significativamente na quantificação dos danos causados por processos erosivos, como as características de revestimento do leito e o sistema de drenagem. Quando da ausência ou mau dimensionamento do primeiro e ineficiência do segundo, propiciam a baixa permeabilidade do solo no leito das estradas e provocam o aumento do escorrimento superficial, propiciando o desenvolvimento de processos erosivos, principalmente quando se trata de condições de relevo mais acidentado, comum em áreas de uso florestal.

Camargo Corrêa et al. (2005) advertem que essa situação se agrava durante a construção das estradas, devido à retirada da cobertura vegetal, à movimentação do solo e à compactação de seu leito, tornando tais vias muito vulneráveis à erosão causada pela chuva, e durante sua utilização, devido ao tráfego pesado. Tais situações também foram referenciadas por autores como Vital et al. (1999), os quais sugerem que, em áreas de uso florestal, as perdas de solo estão associadas principalmente às atividades de exploração florestal e abertura de estradas. Christopher (2002) complementa sugerindo que as maiores perdas de solo ocorrem durante e imediatamente após a construção de estradas, devido à sua instabilidade e aos distúrbios causados pela passagem de caminhões e equipamentos. Fujihara (2002) menciona a existência de várias formas de erosão quanto ao grau de intensidade, e sugere como principais tipos a erosão laminar e a erosão linear, sendo que esta última é causada pela concentração das linhas de fluxo de água de escoamento superficial, resultando em incisões na superfície do terreno em forma de sulcos, ravinas e voçorocas, com consequente solapamento das margens de canal.

Assim sendo, a utilização de modelos numéricos para predição de erosão do solo tem sido uma ferramenta importante para o conhecimento da magnitude dos danos ambientais.

Os primeiros modelos alvitrados tiveram início na década de 40 , com a equação proposta por Zingg (1940), que relacionava as perdas de solo com a declividade e o comprimento da vertente. Outros fatores influentes na evolução desses modelos, como cobertura de solo e práticas conservacionistas, foram acrescentados, buscando-se definir o conceito de limite de perda de solo (BAGNOLD, 1941; ELLISON, 1947; US ARMY/CERL, 1997). Quando acrescentou-se o fator chuva aos fatores de solo e manejo, originou-se a Equação de Musgrave, amplamente utilizada e conhecida (MUSGRAVE, 1947; WISCHMEIER; SMITH, 1978; MEYER; MC'CUNE, 1958; WISCHMEIER, 1959; US ARMY/CERL, 1997). Após a publicação do trabalho de Wischmeier; Smith (1965), a equação passou a ser denominada Universal Soil Loss Equation - USLE (WISCHMEIER; SMIT, 1978; US ARMY/CERL, 1997) e ser utilizada mundialmente, com adaptações regionais, inclusive no Brasil (FERRARI et al., 1996).

A Equação Universal de Perdas do Solo (USLE) e suas derivadas, como a Equação Universal de Perdas do Solo Revisada (RUSLE) e a Equação Universal de Perdas do Solo Modificada (MUSLE), representam modelos empíricos comumente utilizados para predição de perda de solo por erosão (HORSWELL; QUINN, 2003), que compreende um dos mais eficientes modelos de estimativa de perda de solo utilizados no mundo (SOUZA et al., 2003).

Entre os modelos e sistemas de informação geográficos usados para predição de perda de solo citados, alguns vêm sendo testados para estimar erosão em estradas no Brasil, destacando-se os seguintes: WEPP (MACHADO et al., 2003), GRASS (ANTONANGELO, 2004) e a RUSLE (CAMARGO CORREAA, 2005).

Este trabalho teve como objetivo o monitoramento das perdas reais de solo pelo período de um ano, provenientes de estradas secundárias e aceiros, cujos resultados observados foram confrontados com os valores de perdas de solo estimadas através da RUSLE.

\section{MATERIAL E MÉTODOS}

\section{Descrição geral das áreas amostradas}

As áreas avaliadas pertencem a uma empresa florestal localizada no Planalto Norte Catarinense, que possui reflorestamentos com Pinus sp. e Eucalyptus sp. distribuídos no sul do Paraná e norte de Santa Catarina. Essa empresa adota como estratégia de ação o macro e microplanejamento para as atividades de colheita e transporte florestal, aumentando e adaptando sua rede viária em suas Unidades de Manejo Florestal (UMF) à medida que são submetidas à colheita. Essas UMF são diferenciadas entre si em função das características de sítio, produção, manutenção, preservação e colheita. 
Este trabalho foi desenvolvido nas UMF Bugre e Paredão, localizadas no município de Três Barras (SC), onde foram amostrados trechos de aceiros (divisoras) e estradas secundárias, que apresentam diferenciações nas condições de relevo e características edáficas. Essas estradas apresentam revestimento primário com material betuminoso, relevo plano a suave ondulado e solo com textura muito argilosa a argilosa. Segundo Pinto (2005), essas áreas representam $22 \%$ do total das áreas destinadas à produção florestal.

O monitoramento dos trechos de estradas avaliados teve início em março/03 e término em junho/04. Durante esse período, foram coletados semanalmente os sedimentos nas calhas e a água retida nos baldes, juntamente com as informações de precipitação da empresa e através de pluviógrafo instalado nas UMF amostradas.

a) Clima - O clima predominante nas regiões amostradas é do tipo Cfbl: temperado brando, chuvoso com verão fresco, segundo Köepen, com precipitação média anual de $1800 \mathrm{~mm}$, distribuída com maior frequência nos meses de dezembro a março, e em menor quantidade nos meses de junho a agosto. A temperatura média anual é de $18{ }^{\circ} \mathrm{C}$, as médias mínimas alcançadas foram de $7{ }^{\circ} \mathrm{C}$ e as médias máximas foram de $26{ }^{\circ} \mathrm{C}$.

b) Estradas - Foram avaliados trechos de estradas inseridos nas UMF Bugre e Paredão, que apresentavam o sistema viário composto da seguinte forma:

- $\quad 59,81 \mathrm{~km}$ de estradas, com densidade de $23,82 \mathrm{~m} \cdot \mathrm{ha}^{-1}$;

- $\quad 15,10 \mathrm{~km}$ de aceiros internos (divisoras), com densidade de 6,01 m.ha ${ }^{-1}$;

- $\quad 14,81 \mathrm{~km}$ de aceiros externos (contornos), com densidade de 5,90 m.ha ${ }^{-1}$.

As estradas principais e parte das secundárias apresentam revestimento primário constituído de material sedimentar presente na região (folhelho). A largura média encontrada nas estradas secundárias foi de $6 \mathrm{~m}$, e dos aceiros, $4 \mathrm{~m}$.

c) Geologia - As UMF avaliadas apresentam a seguinte descrição geológica, segundo o Mapa Geológico do Estado de Santa Catarina (1986):

- Unidade litoestatigráfica: Subgrupo: Tubarão, Grupo: Guatá/Pp, Formação: Palermo.

- Litologia e ambiente: Seção média: sedimentos marinhos compreendendo siltitos e folhelhos esverdeados com níveis carbonáticos argilosos silicificados em superfície e subordinadamente arenitos muito finos.

- Geomorfologia: formas tabulares, planalto de Canoinhas, formas de cobertura sedimentar. Cobertura fenerozoica não dobrada.

d) Solos - Os solos encontrados nessas UMF pertencem às classes Latossolos, Argissolos, Cambissolos, Gleissolos, Neossolos e Litólicos Aluviais (RIGESA, 2004). No entanto, a área avaliada apresentou solos pertencentes a duas classes de Argissolo.

- Argissolo Vermelho-escuro, Álico, A Moderado, textura argilosa, relevo suave ondulado. Nesse tipo de solo foram instalados os tratamentos que representam os trechos de estrada sem conservação e estradas do povoamento (aceiros ou divisoras) e suas respectivas áreas de contribuição.

- Argissolo Vermelho-escuro Latossólico, Álico, A Proeminente, textura muito argilosa, relevo suave ondulado. Nessa classe de solo foram instalados o tratamento que representa $\mathrm{o}$ trecho de estrada com conservação e sua respectiva área de contribuição. No entanto, a variabilidade textural observada nas amostragens efetuadas nas UMF, oscilou entre muito argilosa a argilosa, com teores de argila entre $700 \mathrm{~g} \cdot \mathrm{kg}^{-1}$ e $500 \mathrm{~g} \cdot \mathrm{kg}^{-1}$, respectivamente.

e) Relevo - As condições de relevo observada nas amostragens das UMF variaram entre plano a suave ondulado, variando de $2 \%$ a $7 \%$ nos tratamentos representados pelos trechos de estrada sem conservação e estradas do povoamento, com suas respectivas áreas de contribuição. Já no trecho de estrada com conservação e sua respectiva área de contribuição, as condições de relevo variaram entre $7 \%$ e $9 \%$. Os trechos avaliados nas UMF Bugre e Paredão localizadas no município de Três Barras encontram-se na figura 1.

\section{Equipamentos e metodologia utilizada}

Para avaliação das perdas de solo e água, utilizou-se calha coletora de solo conhecida como amostrador de enxurrada tipo Coshocton (PARSONS, 1954), que determina o volume de solo e água 
perdidos através de escorrimento superficial e apresenta como característica uma roda com ranhuras na superfície, cujo objetivo é fazê-la girar durante a enxurrada. Parte dos sedimentos grosseiros fica retida na calha e apenas $1 \%$ do volume total de água com sedimentos é coletada, alíquota obtida através de uma fenda (sampling head), que direciona o material amostrado para recipientes coletores (baldes com capacidade de 62 1) conectados através de cano, de acordo com a figura 2, no momento da instalação da calha.

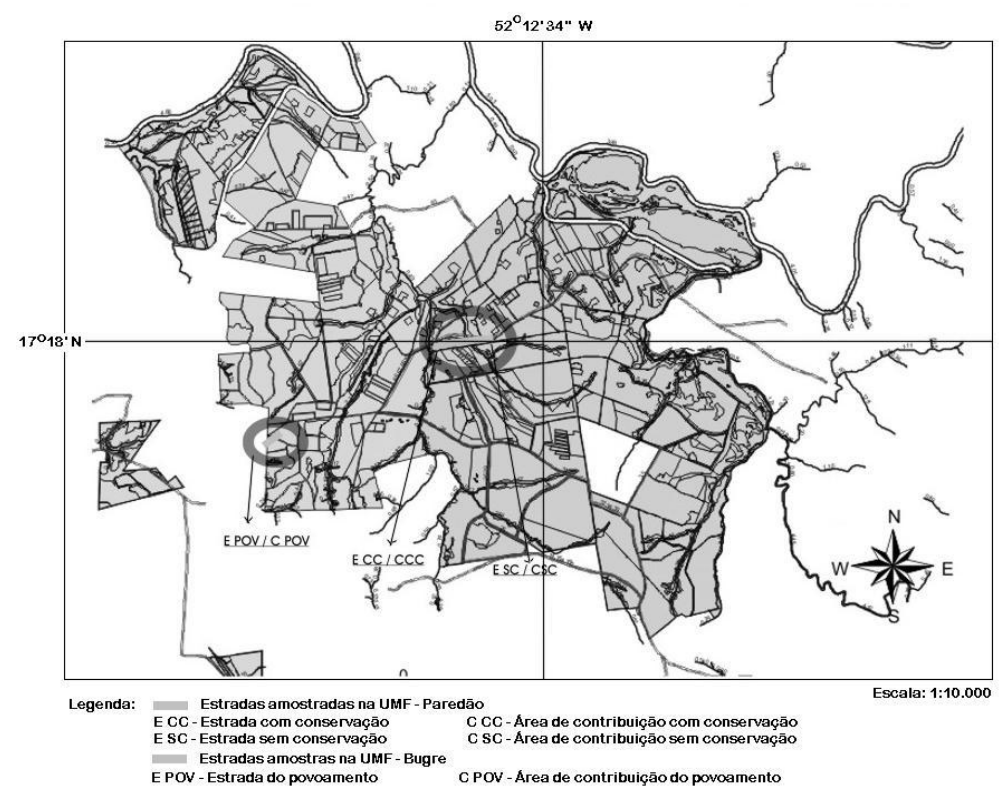

Figura 1. Trechos de estradas florestais avaliados nas UMFs localizadas no município de Três Barras. Figure 1. Parts of forest roads evaluated in FMUs located at Três Barras.

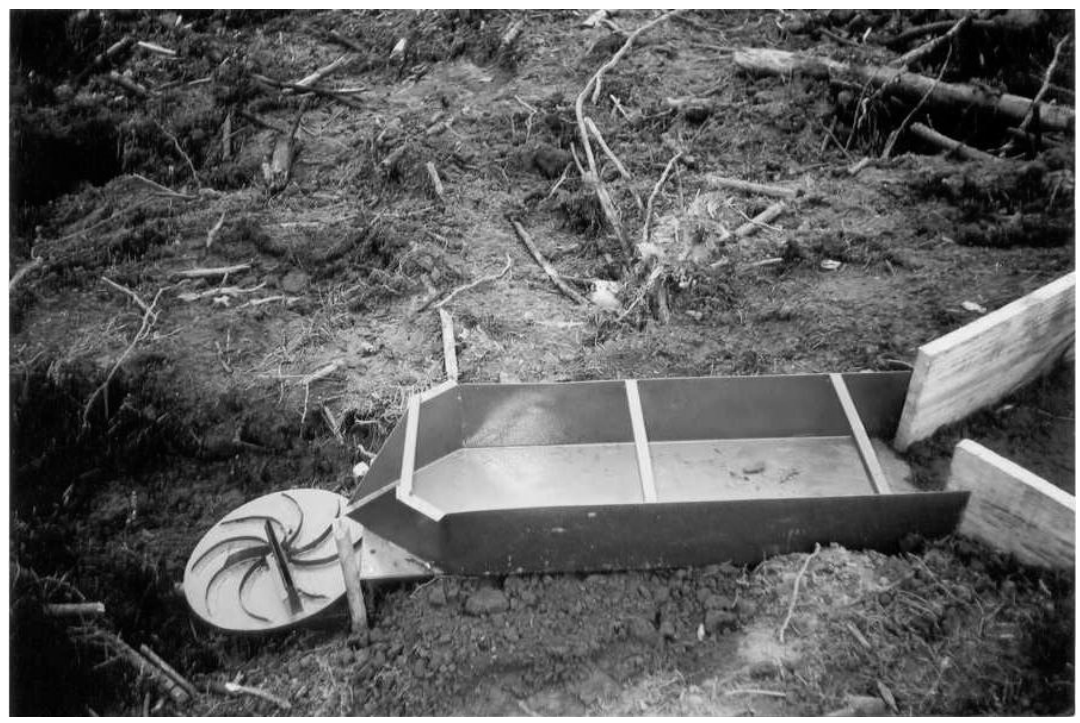

Figura 2. Detalhes da calha coletora de enxurrada Coshocton, no momento da implantação do projeto. Figure 2. Details of Coshocton runoff samplers, in the time of project implantation.

A determinação analítica dos sedimentos retidos nas calhas coletoras se deu através de pesagem 
e avaliação quinzenal durante o período de monitoramento, sendo submetidos à análise de rotina, efetuadas no Laboratório de Fertilidade do Departamento de Solos e Engenharia Agrícola da UFPR. A análise de textura foi determinada através do método da pipeta, no Laboratório de Física do Departamento de Solos e Engenharia Agrícola da UFPR, seguindo a metodologia adotada pela EMBRAPA (1997). As propriedades físicas e químicas das amostras de água coletadas nos baldes foram submetidas à metodologia proposta por Cogo (1978), e as análises foram efetuadas no Laboratório de Pesquisas Hidrogeológicas da UFPR, através dos seguintes métodos de análise instrumental: espectrometria de absorção atômica, espectrometria de emissão e fotocolorimetria. A rugosidade do solo nos trechos de estrada foi determinada através de perfilometria, utilizando-se perfilômetro com barras corrediças. Foram amostrados quatro pontos nos trechos de estrada avaliados, e a tomada desses dados se deu no momento da implantação e no final do projeto. A média das diferenças entre a primeira e a ultima amostragem, considerando os quatro pontos amostrados, representam a rugosidade inicial e final dos trechos amostrados.

\section{Descrição dos tratamentos}

Os tratamentos de estrada avaliados contemplaram dois trechos de estradas secundárias e um trecho de aceiro (divisora). Para cada tratamento foi demarcada uma amostragem de $1 / 2$ ha, que corresponde às áreas de contribuição adjacentes aos trechos de estradas avaliados. As características das amostragens são apresentadas na tabela 1 .

Tabela 1. Legenda dos tratamentos avaliados nas Unidades de Manejo Florestal (UMF).

Table 1. Treatment legend evaluated in Forest Management Units (FMU).

\begin{tabular}{lccc}
\hline Tratamento & Município & Legenda & Declividade (\%) \\
\hline Estrada com Conservação & Três Barras & TB/E/CC & 2 \\
Estrada sem Conservação & Três Barras & TB/E/SC & 7 \\
Estrada Povoamento & Três Barras & TB/E/POV & 3 \\
Contribuição com Conservação & Três Barras & TB/C/CC & 7 \\
Contribuição sem Conservação & Três Barras & TB/C/SC & 8 \\
Contribuição Povoamento & Três Barras & TB/C/POV & 9 \\
\hline
\end{tabular}

a) Estradas

(E): trechos de estrada.

(E CC): trechos que apresentaram medidas de conservação adotadas pela empresa, representadas por barreiras de contenção da enxurrada, associadas às valas de retenção de água, equidistantes em aproximadamente 50 metros. Dessa forma, cada parcela apresentou três barreiras de contenção de enxurrada, sendo uma em cada extremidade da parcela e uma central, todas associadas à vala de retenção de água. Trecho de estrada sem conservação.

(E SC): trechos de estradas que não apresentaram medidas de conservação e trechos de estrada do povoamento.

(E POV): corresponde a trechos de aceiros ou divisoras.

As dimensões das parcelas foram de 100 metros de comprimento de estrada com largura média de 6 metros, sendo delimitadas por linhas de drenagem nas laterais das estradas e por barreiras de contenção nas extremidades. Essas parcelas foram protegidas por lonas pretas nas laterais, a fim de evitar a deposição de sedimentos provenientes das suas respectivas áreas de contribuição, assim como as barreiras de contenção de enxurrada, evitando o acréscimo de sedimentos oriundos de outros segmentos da estrada. Na porção mais baixa da parcela, seguindo a linha de drenagem da estrada, foram instaladas as calhas coletoras de solo.

b) Áreas de Contribuição (C): representam áreas adjacentes às estradas, demarcadas a partir da porção mais alta da paisagem e que apresentaram inclinação direcionada para as estradas equivalentes, com dimensões de 50 x $100 \mathrm{~m}(1 / 2 \mathrm{ha})$. Essas áreas foram delimitadas por linhas de drenagem e, na porção mais baixa da paisagem, foram instaladas as calhas coletoras de solo. Esses tratamentos contemplaram novos plantios, que haviam passado por preparo convencional do solo utilizado pela empresa, que consiste em enleiramento de resíduos e preparo do solo com subsolador morro abaixo. A área de contribuição do 
povoamento (C POV) contempla a área adjacente ao trecho de estrada no povoamento (E POV), demarcada dentro de um povoamento de Pinus taeda, com idade de 6 anos.

\section{Equação de perdas de solo}

A equação utilizada para estimar as perdas de solo foi a Revised Universal Soil Loss Equation (RUSLE), também conhecida como Equação Universal de Perdas de Solo (EUPS), sendo definida através da seguinte equação:

$$
\mathrm{A}=\mathrm{R} \cdot \mathrm{K} \cdot \mathrm{L} \cdot \mathrm{S} \cdot \mathrm{C} \cdot \mathrm{P}
$$

Em que: $\mathrm{A}=$ perda de solo calculada por unidade de área, em t.ha ${ }^{-1} \cdot \mathrm{ano}^{-1}$.

$\mathrm{R}=$ fator erosividade da chuva, índice de erosão pela chuva, em MJ. $\mathrm{mm} \cdot \mathrm{ha}^{-1} \cdot \mathrm{h}^{-1}$

$\mathrm{K}=$ fator erodibilidade do solo, em MJ . $\mathrm{mm} \cdot \mathrm{ha}^{-1} \cdot \mathrm{h}^{-1}$

$\mathrm{L}=$ fator comprimento de rampa, valores medidos em metros de rampa (100 metros).

$\mathrm{S}=$ fator declividade, baseado nos valores em $\%$, da declividade.

$\mathrm{C}=$ fator uso e manejo do solo.

$\mathrm{P}=$ fator prática conservacionista aplicada (fatores antrópicos e adimensionais).

$\mathrm{O}$ fator $\mathrm{R}$ foi determinado em função de histórico diário de precipitação, com informações de intensidade de chuvas decorrentes do período de março de 2003 a junho de 2004 . $\mathrm{O} \mathrm{EI}_{30}$ foi estimado através das equações apresentadas a seguir, propostas por Wischmeier; Smith (1959).

Definição da energia cinética, através da equação 1.

$$
\mathrm{E}=0,119+0,0873 \log \mathrm{I}
$$

Em que:

$$
\mathrm{E}=\text { energia cinética por } \mathrm{mm} \text { de chuva }\left(\mathrm{MJ} \mathrm{ha}^{-1} \cdot \mathrm{mm}^{-1}\right) \text {. }
$$

$\mathrm{I}=$ intensidade de chuva $\mathrm{em} \mathrm{mm} \cdot \mathrm{h}^{-1}$.

O índice de erosividade da chuva foi obtido através da multiplicação da energia cinética calculada pela intensidade máxima de chuva em 30 minutos, expresso em MJ.mm ha ${ }^{-1} \cdot \mathrm{h}^{-1} \cdot \mathrm{ano}^{-1}$, conforme equação 2 .

$$
\mathrm{EI}_{30}=\mathrm{E} . \mathrm{I}_{30}
$$

A determinação do fator R é dada em função de um histórico de chuvas. Neste estudo, esse fator foi estimado através da soma dos valores mensais do índice de erosividade, conforme equação 3.

$$
R=\sum_{J=1}^{12} \mathrm{EI}_{30}
$$

O fator K foi calculado com base na equação 4, proposta por Dissmeyer; Foster (1980).

$$
\mathrm{K}=2,1 \mathrm{M}^{1,14} \cdot\left(10^{-6}\right) \cdot(12-\mathrm{a})+0,0325 \cdot(\mathrm{b}-2)+0,025(\mathrm{c}-3)
$$

Em que:

$\mathrm{K}=$ erodibilidade do solo (t.h. $\left.\mathrm{Mj}^{-1} \cdot \mathrm{mm}^{-1}\right)$.

$\mathrm{M}=(\%$ silte + areia fina $) .(100-\% \mathrm{C}) \quad \mathrm{a}=\%$ de carbono

$\mathrm{b}=$ estrutura.

$\mathrm{c}=$ classe de permeabilidade.

$1=$ muito fina granular.

$6=$ muito lenta.

$2=$ fina granular.

$5=$ lenta.

3 = média a grossa granular.

4 = blocos, laminar ou maciça.

$4=$ lenta para moderada.

$3=$ moderada

2 = moderada para rápida.

$1=$ rápida.

Para cálculo dos fatores L e S foi utilizada a equação 5, proposta por Bertoni; Lombardi Neto (1999).

$$
\mathrm{LS}=0,00984 \cdot \mathrm{C}^{0,63} \cdot \mathrm{D}^{1,18}
$$


subfatores.

O fator $\mathbf{C}$ foi determinado através equação 6 , que representa a multiplicação dos seguintes

$$
\text { C }=\text { CC. BS . SR . SC . RB . DE . CT }
$$

A composição dos subfatores foi definida da seguinte forma:

- Cobertura de Dossel ou Solo Descoberto (CC): Nesse subfator, ficou estipulado o valor de 1 para todos os tratamentos, considerando-se que não há cobertura de dossel nas estradas e que as suas respectivas áreas de contribuição foram submetidas a corte raso. Nas áreas adjacentes ao trecho de estrada do povoamento, onde havia cobertura de dossel, não foram constatadas perdas de solo durante o período.

- Cobertura da Superfície ou Cobertura por Resíduo (BS): Os valores desse subfator foram estipulados através de Dissmeyer; Foster (1980) e o valor atribuído a esse subfator foi de 0,03, considerando-se aproximadamente $50 \%$ da área com solo descoberto.

- Rugosidade Superficial do Solo (SR): Nesse subfator, utilizou-se a equação 7, proposta por Renard et al. (2000):

$$
\mathrm{SR}=\exp { }^{[-0,66(\mathrm{Ru}-0,24)]}
$$

Os valores de rugosidade foram obtidos através de perfilometria, e os resultados obtidos estão apresentados na tabela 2 .

- Reconsolidação do Solo (SC): Esse subfator foi determinado através de Dissmeyer; Foster (1980), e os valores estipulados para os trechos de estrada avaliados foram de 0,622 e 0,245 para as áreas de contribuição em ambas as condições avaliadas.

- Agregação Residual do Uso Anterior (RB): Esse subfator foi proposto através de Dissmeyer; Foster (1980), tendo sido estabelecidos os valores de 0,9 para os trechos de estrada e 0,4 para as áreas de contribuição.

- Efeito de Degraus (DE): Na tabela 2 estão os valores encontrados para esse subfator, de acordo com Dissmeyer; Foster (1980).

A soma dos subfatores que compõem o fator C são apresentados na tabela 2.

$\mathrm{O}$ fator $\mathrm{P}$ representa o efeito da prática conservacionista. Como nas áreas de contribuição o preparo de solo foi feito morro abaixo, o valor estipulado para esse fator foi 1 nessas áreas. Já nos trechos de estrada avaliados, a definição desse fator se deu em função das perdas de solo por tratamento e as perdas totais dos tratamentos em estradas. Dessa forma, foram obtidos os seguintes valores para esse fator: 0,4 para os tratamentos de estrada com conservação, 0,5 para os trechos de estrada sem conservação e 0,1 para os trechos de estradas no povoamento nas UMF amostradas.

Os resultados de perdas de sedimentos por processos erosivos, obtidos durante o monitoramento, foram submetidos à análise estatística de Regressão Linear através do programa computacional STATISTICA. Os resultados de perdas totais avaliadas foram confrontados com os estimados através da RUSLE e submetidos à análise estatística através do Teste $\mathrm{t}$, por médias pareadas usando o programa Excel.

\section{RESULTADOS E DISCUSSÃO}

\section{Estimativas de perda através da $R U S L E$}

As perdas reais de solo avaliadas nos trechos de estrada e suas respectivas áreas de contribuição foram equiparadas com os resultados obtidos através da RUSLE, cujos fatores apresentaram os resultados a seguir comentados.

O fator R, que representa a erosividade da chuva, apresentou o valor de $8.831,8 \mathrm{MJ} \cdot \mathrm{mm} \cdot \mathrm{ha}^{-1} \cdot \mathrm{h}^{-1}$, considerando que as chuvas acumuladas no período de avaliação representaram 1.453,9 mm. Alguns estudos sobre erosividade da chuva desenvolvidos pela UDESC (Universidade do Estado de Santa Catarina) apontaram valores médios para erosividade da chuva em Santa Catarina. Bertol (1993) determinou o índice de erosividade através do $\mathrm{EI}_{30}$ para o município de Lages (SC), obtendo um valor médio anual de 5.694 MJ.mm.ha ${ }^{-1} \cdot \mathrm{h}^{-1}$.ano ${ }^{-1}$, e para a localidade de Campos Novos (SC), no período de 1981 a 1990 , também com o índice $\mathrm{EI}_{30}$, obtendo um índice médio anual de $6.329 \mathrm{MJ} \cdot \mathrm{mm} \cdot \mathrm{ha}^{-1} \cdot \mathrm{h}^{-1} \cdot \mathrm{ano}^{-1}$ (BERTOL, 1994). 
$\mathrm{O}$ fator $\mathrm{K}$, que representa a erodibilidade dos solos, foi calculado de acordo com a equação 4, variando de 0,022 a 0,023 t.h. $\mathrm{MJ}^{-1} \cdot \mathrm{mm}^{-1}$, para Argissolos Vermelho-escuros avaliados nas UMF. O fator erodibilidade do solo apresenta variabilidade espacial e temporal bastante significativa, que pode ser parcialmente explicada pelas interferências climáticas que afetam o potencial erosivo das chuvas, podendo também ser fortemente influenciada pelo teor de água do solo antecedente às chuvas e pela variabilidade do solo. Essas características associadas influenciarão na suscetibilidade do solo aos processos erosivos.

Os fatores $\mathrm{L}$ e $\mathrm{S}$, que representam o comprimento e a inclinação de rampa respectivamente, foram calculados através da equação 5, e os resultados obtidos encontram-se na Tabela 2, assim como os valores obtidos para o fator $\mathrm{C}$, calculado através da equação 6 . Alguns modelos de predição de processos erosivos, como o GRASS (ANTONANGELO, 2005), consideram apenas os fatores L e S, para a determinação de erosão em estradas. Os demais fatores, como erosividade da chuva e erodibilidade do solo, não são referenciados.

Tabela 2. Valores calculados dos subfatores rugosidade de superfície (SR) e efeito de degraus (DE) e dos fatores C e LS, utilizados, nas UMFs de Três Barras (SC).

Table 2. Sub-factors calculated values from superficial roughness and degrees effect and factors $\mathrm{C}$ and LS, utilized at Três Barras (SC) FMUs.

\begin{tabular}{lccccccccccc}
\hline \multirow{2}{*}{ Tratamento } & \multicolumn{2}{c}{ Subfatores } & Fator & Fator & Fator & \multirow{2}{*}{ Tratamento } & \multicolumn{2}{c}{$\begin{array}{c}\text { Subfatores } \\
\text { SR }\end{array}$} & $\begin{array}{c}\text { Fator } \\
\text { DE }\end{array}$ & $\begin{array}{c}\text { Fator } \\
\text { L e S }\end{array}$ & $\begin{array}{c}\text { Fator } \\
\text { P }\end{array}$ \\
\hline 3B E CC & 0,957 & 0,99 & 0,05 & 0,41 & 0,4 & 3B C CC & 0,277 & 0,59 & 1,15 & 0,0005 & 1 \\
3B E SC & 0,614 & 0,67 & 0,02 & 1,78 & 0,5 & 3B C SC & 0,410 & 0,49 & 1,35 & 0,0006 & 1 \\
3B E POV & 0,613 & 0,99 & 0,03 & 0,65 & 0,1 & 3B C POV & 0,643 & - & 1,55 & - & 1 \\
\hline
\end{tabular}

Comparativo entre as perdas reais de solo e estimativas através da $R U S L E$

Os resultados de perda de solo obtidos nas UMF foram equiparados aos valores estimados através da RUSLE e os resultados estão descritos na tabela 3. As estimativas obtidas superestimaram os valores reais em todos os tratamentos avaliados nessas UMF.

A densidade de estradas é referenciada na literatura em metros lineares por hectare, portanto, todos os valores de perda de solo em estradas citados nesse trabalho foram expressoz em toneladas por quilômetro de estrada $\left(\mathrm{t} . \mathrm{km}^{-1}\right)$. Já as áreas de contribuição, por representarem áreas de plantio, estão expressas em toneladas por hectares $\left(\mathrm{t} . \mathrm{ha}^{-1}\right)$.

Considerando-se apenas os valores de perdas reais de solo, a ocorrência de processo erosivo em estradas foi responsável por perdas de solo na ordem de $99,7 \%$ das perdas totais de solo, evidenciando a importância de um bom planejamento de estradas, que atenda as necessidades da propriedade, mas que prime pela menor densidade possível, a fim de reduzir a possibilidade de ocorrência de processos erosivos.

Tabela 3. Estimativa de perda de solo através da RUSLE e as perdas reais de solo avaliadas nas UMF de Três Barras.

Table 3. Soil losses estimative through RUSLE and real soil losses evaluated in Três Barras FMU.

\begin{tabular}{lccc}
\hline \multirow{2}{*}{ Tratamentos } & \multicolumn{3}{c}{ UMF/Três Barras } \\
\cline { 2 - 4 } & Perda estimada & Perda real acumulada & Diferença de estimativa (\%) \\
\hline E/CC & 1,66 & 1,44 & $+15,3$ \\
E/SC & 4,12 & 2,40 & $+71,7$ \\
E/POV & 0,43 & 0,14 & $+207,1$ \\
Subtotal & 6,21 & 3,98 & $+56,0$ \\
C/CC & 0,11 & 0,010 & $+100,0$ \\
C/SC & 0,16 & 0,004 & $+3.900,0$ \\
C/POV & - & - & - \\
Subtotal & 0,27 & 0,014 & $+1.828,6$ \\
\hline Total & 6,48 & 3,99 & $+62,4$ \\
\hline
\end{tabular}

Os valores de perdas de solo nas estradas estão apresentados em t.km ${ }^{-1}$, e nas áreas de contribuição, em t.ha ${ }^{-1}$, nas duas regiões amostradas. 
Outra evidência constatada neste estudo se refere às medidas de conservação adotadas pela empresa, que consistem na implantação de water bar (barreiras de contenção de enxurrada), associada a valas de retenção de sedimentos, equidistantes em $50 \mathrm{~m}$, que representaram uma redução na ocorrência de processos erosivos em torno de $24 \%$, quando comparados os tratamentos com e sem medida de conservação.

Nas perdas totais de solo provenientes de estradas, constatou-se que estradas sem medidas de conservação são responsáveis por perdas de solo na ordem de 60,3\%. Já no tratamento que contempla as medidas de conservação adotadas pela empresa, as perdas representaram $36,2 \%$, e as perdas procedentes de aceiros representaram $3,5 \%$ neste estudo.

No que se refere às perdas globais de solo avaliadas, foi constatado que as perdas procedentes de áreas de contribuição foram da ordem de $0,35 \%$, sendo que não foram constatadas perdas de solo em áreas com plantio de Pinus taeda, o que pode ser atribuído aos diversos fatores que interferem no processo de erosão, entre os quais se destacam a cobertura de dossel, em função do fechamento das copas e a cobertura do solo pela serapilheira, que intercepta o efeito das gotas no solo, evitando a desagregação deste e dificultando o processo de transporte de sedimentos, uma vez que a maior parte da água proveniente da chuva é percolada no perfil do solo.

Ao compararmos os valores reais e globais de perda de solo com os valores estimados através da RUSLE, através da análise estatística pelo Teste t pelo programa Excel, foram constatados os seguintes resultados, considerando significância estatística de 95\%: $\mathrm{N}=7$, Correlação de Pearson $\mathrm{R}=0,9924$, valor $\mathrm{t}=1,91, \mathrm{t}$ crítico unicaudal $=1,94, \mathrm{t}$ crítico bicaudal $=2,45$.

Quando analisados separadamente os tratamentos de estradas de suas respectivas áreas de contribuição, foram obtidos os seguintes resultados da análise estatística:

$\mathrm{N}=3$, Coeficiente de Pearson $\mathrm{R}=0,9619$, valor $\mathrm{t}=1,52, \mathrm{t}$ crítico unicaudal $=2,92, \mathrm{t}$ crítico bicaudal $=$ 4,30 .

Nas áreas de contribuição, os resultados obtidos foram: $\mathrm{N}=3$, Coeficiente de Pearson $\mathrm{R}=$ 0,5825 , valor $\mathrm{t}=1,87, \mathrm{t}$ crítico unicaudal $=2,92, \mathrm{t}$ crítico bicaudal $=4,30$.

A alta correlação entre os valores reais e estimados através da equação, constatada através da análise estatística, evidencia o potencial de uso da RUSLE na predição de perdas de solo por processos erosivos em estradas, porém há de se formar um banco de dados de informações sobre os fatores que contemplam a equação.

\section{CONCLUSÕES}

- As perdas de solo provenientes de estradas de uso florestal representaram cerca de $99,7 \%$ das perdas totais de solo, nas condições avaliadas.

- As medidas de conservação adotadas pela empresa, para a conservação de estradas, reduziram as perdas de solo em torno de $25 \%$.

- Os valores de perdas de solo estimados através de RUSLE proporcionaram bom ajuste estatístico, além de alta correlação com os valores reais de perda de solo, para as UMFs avaliadas.

- A equação superestimou as perdas de solo em estradas com essas características de solo e relevo.

- A equação apresenta potencial de uso para predição de perdas de solo por erosão em estradas, no entanto há necessidade de formulação de banco de dados dos fatores que a compõem, para ajustes.

- A erosividade da chuva (fator R) é determinante na ocorrência de processos erosivos, e os fatores L e $\mathrm{S}$, que descrevem o comprimento e a declividade da rampa, associados ao fator $\mathrm{K}$, que representa a erodibilidade do solo, estão diretamente relacionados com o volume de sedimentos provenientes de estradas observado durante o período.

\section{REFERÊNCIAS}

ANTONAnGELO, A. Avaliação do Sistema de Informações Geográficas GRASS para Predição dos Riscos de Erosão em Estradas de Uso Florestal. 100 p. Tese (Doutorado) - Universidade Estadual Paulista, Botucatu, 2004. 
BAGNOLD, R. A. The physics of blown sand and desert dunes. London: Methuen \& Co. 1941. 265 p.

BERTONI, J.; LOMBARDI NETO, F. Conservação do solo. São Paulo: Ícone,1999. 392 p.

BERTOL, I. Índice de erosividade (EI30) para Lages (SC) - 1a aproximação. Pesquisa Agropecuária Brasileira, Brasília, DF, v. 28, n. 4, p. 515-521, abr. 1993.

BERTOL, I. Erosão hídrica em Cambissolo Húmico distrófico sob diferentes preparos do solo e rotação de culturas. Revista Brasileira de Ciência do Solo, Campinas, v. 18, p.267-271, 1994.

BORTOLOZZO, A. R.; SANS L. M. A. Um paralelo entre USLE e suas novas versões. Revista FactuCiência, Unaí/MG, n. 1, p. 1951 -1958, jan./jun. 2001.

CAMARGO CORRÊA, C. M.; ROLOFF, G.; MALINOVSKI, J. R. Parameters of Quality of the Water Originating from Forest Roads in Brazil. In: SOIL AND WATER CONSERVATION SOCIETY, 2005 Annual Conference. Rochester/NY. CD. 2005. Soil and Water Conservation Society. 2005.

CAMARGo CORREAA, C. M. Perdas de solo e a qualidade da água procedente de estrada de uso florestal no Planalto Catarinense. 155 f. Tese (Engenharia Florestal) - Setor de Ciências Agrárias, Universidade Federal do Paraná, Curitiba, 2005.

CHRISTOPHER Jr., E. A. Post Harvest evaluation of Best Management Practices for the Prevention of Soil Erosion in Virginia. 125 p. Master of Science in Forestry. Virginia Polytechnic Institute and State University. Blacksburg. Virginia. April 2002.

COGO, N. P. Uma contribuição à metodologia de estudo das perdas de erosão em condições de chuva natural. I. Sugestões gerais, medições dos volumes, amostragem e quantificação de solo e água da enxurrada ( $1^{\mathrm{a}}$ aproximação). In: ENCONTRO NACIONAL DE PESQUISA SOBRE CONSERVAÇÃO DO SOLO. 2., 1978, Passo Fundo. Anais... Passo Fundo. EMBRAPA. CNPT, 1978. p. 75-98.

CONSORT, J. J.; ZALUSKI, M. H.; ANTONIOLI, S. B. Soil erosion and Deposition Modeling using Arc GIS ${ }^{\mathrm{TM}}$. In: ASTC - ADVANCED SIMULATION TECHNOLOGIES CONFERENCE. Session 5: Environmental Technology I. Arlington, v. 18, 21 abr. 2004.

DISSMEYER, G. E.; FOSTER, G. R. A guide for predicting sheet and rill erosion on forest land. Asheville, N. C.: Southeastern Forest Experiment Station and Southeastern Area, State \& Private Forestry Atlanta, 1980. (Technical Publication SA-TP-11).

ELLISON, W. D. Soil erosion. Soil Science Society American Proceddings. Madison, v. 12, p. 479484, 1947.

EMPRESA BRASILEIRA DE PESQUISA AGROPECUÁRIA (EMBRAPA). Serviço Nacional de Levantamento de Conservação de Solos. Manual de Métodos de Análise de Solos. Rio de Janeiro, 1997. v. 1 .

FERRARI, J. A.; HIRUMA, S. T.; ANTONIETTI, E.; PEREIRA, P. R. B.; TAVARES, R. Mapeamento da susceptibilidade à erosão na Bacia do Rio Quilombo/SP. [S.1.]: Instituto Geológico, 1996. 56 p. (Boletim, 12).

FUJIHARA, A. K. Predição de erosão e capacidade de uso do solo numa microbacia do oeste paulista com suporte de geoprocessamento. 136 p. Dissertação (Mestrado em Ciência Florestal) Escola Superior de Agricultura Luiz de Queiroz, Universidade de São Paulo, Piracicaba, 2002.

HORSWELL, M.; QUINN, N. Minimising sediment delivery to rivers: a spatial modelling approach developed for commercial forestry. DIFFUSE POLLUTION CONFERENCE DUBLIN, 2003. 5 A FOREstry.

MACHADO, C. C.; GARCIA, A. R.; SILVA, E.; FONTES, A. M. Comparação de Taxas de Erosão em Estradas Florestais Estimadas pelo Modelo Wepp (Water Erosion Prediction Project) Modificado em Relação a Medições Experimentais. Revista Árvore, Viçosa, MG, v. 27, n. 3, p. 295-300, 2003. 
MAPA GEOLÓGICO DO ESTADO DE SANTA CATARINA, Ministério das Minas e Energia. Secretaria da Ciência e Tecnologia, Minas e Energia. Coordenadoria dos Recursos Minerais, 1986.

MEYER, L. D.; MC'CUNE, D. L. Rainfall simulator for runoff plots. Agricultural Engineering, Saint Joseph, v. 39, p. 644-648, 1958.

MUSGRAVE, G. W. A quantitative evaluation of factors in water erosion - A first aproximation. Journal of Soil and Water Conservation, Ankeny, v. 2, p. 133-138, 170. 1947.

PARSONS, D. A. Coshocton: type runoff samplers. Laboratory investigations. United States Departament of Agriculture. Soil Conservation Service. Washington, DC, 25 april, 1954. 16 p.

PINTO, L. M. Relatório sumários de avaliação do sistema d manejo florestal da RIGESA Celulose Papel e Embalagens Ltda nos estados de Santa Catarina e Paraná: Norma de Referencia: NBR 14.789:2001. [S.1.], 2005. 52 p.

RENARD, K. G.; FOSTER, G. R.; WEEISIES G. A.; McCOOL. D. K.; YODER, D. C. (Coords.). Predicting soil erosion by water: a guide to conservations planning with the revised soil loss equation (RUSLE). United States: Dept. of Agriculture, 1997. 404 p. (Agric. Handbook, n. 703).

RIGESA, 2004. Levantamento de Solos da Empresa. Documento interno.

SOUZA, C. K.; MARQUES JUNIOR, J.; MARTINS FILHO, M. V.; PEREIRA, G. T. Influência do relevo e erosão na variabilidade espacial de um Latossolo em Jaboticabal "SP". Revista Brasileira de Ciências do Solo. Viçosa, MG, v. 27, n. 6, p. 1067-1074. 2003.

UNITED STATES - US Army / CERL. Army corps of engineers. Construction Engineering Research Laboratories. Evaluation of Technologies for Addressing Factors Related to Soil Erosion on DOD Lands. 1997. (Technical Report. 97/134).

VITAL, A. R. T.; LIMA, W. P.; CAMARGO, F. R. A. Efeitos do corte raso de plantações de Eucalptus sobre o balanço hídrico, a qualidade da água e as perdas de solo e de nutrientes numa microbacia no Vale do Paraíba. SP. Scientia Forestalis, Piracicaba, n. 55, p. 5-16, jun. 1999.

WISCHMEIER, W. H. A rainfall erosion index for a Universal Soil Loss Equation. Soil Science American Proceddings. Madison, v. 23, n. 2, p. 246-249, 1959.

WISCHMEIER, W. H.; SMITH, D. D. Predicting rainfall erosion losses; a guide to conservaion planting. Washington: USDA, 1978. 58 p. (Agriculture Handbook, 537).

ZINGG, A. W. Degree and length of land slope as it effects soil loss in runoff. Agricultural Engineering, Saint Joseph, n. 21, p. 59-64, 1940. 